\title{
Interactive comment on "Volatile organic compounds and ozone air pollution in an oil production region in northern China" by Tianshu Chen et al.
}

Tianshu Chen et al.

xuelikun@sdu.edu.cn

Received and published: 25 March 2020

Pls see the detailed responses to referee's comments in the attachment.

Please also note the supplement to this comment: https://www.atmos-chem-phys-discuss.net/acp-2019-722/acp-2019-722-AC1supplement.pdf 2019. 